\title{
Application value of CT and MRI in diagnosis of primary brain lymphoma
}

\author{
YONGCHUN QIN ${ }^{1 *}$, AIHUA BAO $^{2 *},{\text { HONGJUN } \mathrm{LI}^{3}, \mathrm{XIN} \mathrm{WANG}^{4}, \mathrm{GE} \mathrm{ZHANG}^{5} \text { and JIAFENG ZHU }}^{6}$ \\ ${ }^{1}$ Department of Imaging, People's Hospital of Rizhao, Rizhao, Shandong 276800; ${ }^{2}$ Department of Imaging, \\ Qingdao Women and Children's Hospital, Qingdao, Shandong 266000; ${ }^{3}$ EEG Room, Zhangqiu District Hospital of TCM; \\ ${ }^{4}$ Magnetic Resonance and ${ }^{5}$ Department of Obstetrics, People's Hospital of Zhangqiu, Jinan, Shandong 250000; \\ ${ }^{6}$ Department of Interventional Medicine, People's Hospital of Rizhao, Rizhao, Shandong 276800, P.R. China
}

Received December 22, 2017; Accepted February 21, 2018

DOI: $10.3892 / 01.2018 .8404$

\begin{abstract}
This study explored the correlation between computed tomography (CT) and magnetic resonance imaging (MRI) manifestations and pathological features of primary brain lymphoma to improve the diagnostic accuracy. A total of 230 patients with primary brain lymphoma admitted to People's Hospital of Rizhao from July, 2005 to December, 2016 were selected into the study and their clinical data were analyzed retrospectively. Among them, 87 patients were examined by CT, 74 patients by MRI, 69 patients by both MRI and CT. Features of MRI and CT scanning figures were observed with a focus on the density, number and margins of the lesions, and the diagnostic accuracy was analyzed. A total of 353 lesions were identified from 230 primary brain lymphoma patients, of which 224 were single lesions, and 129 were multiple lesions. Most lesions were on the upper curtain (81.3\%, 187 cases) and 43 cases (18.7\%) were on the lower curtain. Lesion signal of $\mathrm{CT}$ and MRI plain scan showed uniform state, and enhanced scan showed significantly enhanced signal. Diagnostic accuracy of $\mathrm{CT}$ was $82.8 \%$, and sensitivity and specificity was 75.5 and $67.4 \%$, respectively. Diagnostic accuracy of MRI was $83.8 \%$, and sensitivity and specificity was 79.3 and $64.9 \%$, respectively. Diagnostic accuracy of MRI combined with CT was $89.9 \%$, and sensitivity and specificity was 86.3 and $75.8 \%$, respectively. CT combined with MRI can provide better diagnosis for primary brain lymphoma compared with CT or MRI alone, but pathological test is still needed.
\end{abstract}

Correspondence to: Dr Jiafeng Zhu, Department of Interventional Medicine, People's Hospital of Rizhao, 126 Taian Road, Rizhao, Shandong 276800, P.R. China

E-mail: jiafeng_zhu01@163.com

${ }^{*}$ Contributed equally

Key words: computed tomography, magnetic resonance imaging, primary brain lymphoma, application, diagnosis

\section{Introduction}

Primary brain lymphoma is a rare central nervous system malignancy, and only accounts for $1.5 \%$ of all intracranial tumors (1). However, with the continuous development of medical technology in recent years, the widespread use of immunological agents and the maturity of organ transplantations have made patients prone to primary brain lymphoma due to immunodeficiency (2,3). Ferreri et al (4) reported that the number of patients with primary brain lymphoma increased by 80,000 in 2000-2005 compared with that in 1990s. Nayak et al (5) also reported that the incidence of primary brain lymphoma showed an increasing trend, and it will become a type of malignant intracranial tumor with extremely high incidence in 2025. Clinically, prevention of primary brain lymphoma has become an extremely important issue (6). The universal treatment of intracranial tumors is 'early detection and early treatment', and the main clinical diagnosis methods are magnetic resonance imaging (MRI), and computed tomography (CT). Primary brain lymphoma show no obvious signs during the early stages, and specific imaging features are also lacking. Thus, early diagnosis is difficult $(7,8)$. Therefore, this study aimed to analyze the imaging diagnosis of MRI and CT for patients with primary brain lymphoma with an expectation of providing reference and guidance for the early diagnosis of patients with primary brain lymphoma.

\section{Patients and methods}

General information. A total of 230 patients with primary brain lymphoma admitted to People's Hospital of Rizhao (Rizhao, China) were selected from July, 2005 to December, 2016 into the study and their clinical data were analyzed retrospectively. There were 147 males and 83 females, with a mean age of $48.12 \pm 10.57$ years. Among those patients, 87 patients were examined by CT, 74 patients by MRI, and 69 patients by both MRI and CT. Inclusion criteria: Patients with primary brain lymphoma confirmed by biopsy; patients with complete clinical record; patients willing to cooperate with researchers. Exclusion criteria: Patients combined with upper respiratory tract diseases; patients combined with lower gastrointestinal 
diseases; patients combined with cardiovascular diseases; patients transferred to other hospitals during treatment; patients with severe physical disability; patients treated with drugs prescribed by other hospitals. All patients signed written informed consent. The study was approved by the Ethics Committee of People's Hospital of Rizhao. Signed written informed consents were obtained from the patients and/or guardians.

Instruments and methods. CT detector was a LightSpeed 16-slice spiral CT (GE Healthcare Life Sciences, Waukesha, WI, USA), and MRI detector was a Vision Plus 1.5T superconducting scanner (Siemens, Munich, Germany). All reagents used are original equipment supporting reagents. All patients who underwent $\mathrm{CT}$ were subjected to head plain scanning and enhanced CT scanning. Both T1W1 and T2W1 scanning were performed in MRI by using both selective echo (SE) and fast spin echo (FSE) imaging (Table I for parameter settings).

Imaging analysis. Clinical data and pathological test results were used as gold standard to evaluate the accuracies of three methods. Imaging features of lesions in MRI and CT scanning were observed with a focus on the density, number and margins of lesions. All MRI and CT images were analyzed by four experienced imaging physicians through double-blind method, and clinical values were analyzed.

Statistical analysis. SPSS 22.0 statistical software (IBM Corp., Armonk, NY, USA) was used for all statistical analyses. Enumeration data were expressed as rate, and comparisons among multiple groups were performed by Chi-square test. Sensitivity and specificity of diagnosis were analyzed by ROC curve.

\section{Results}

Clinical patient data. Comparison of clinical data showed that there were no significant differences in age, sex, ethnicity, marital status, living area, smoking index, alcohol consumption, exercise status, pathological stages and clinical symptoms among three groups (CT, MRI and CT combined with MRI; p>0.05) (Table II).

Imaging results. A total of 353 lesions were found in 230 primary brain lymphoma patients, and 224 lesions were single lesions, and 129 lesions were multiple lesions. Most lesions were on the upper curtain $(81.3 \%, 187$ cases) and 43 cases $(18.7 \%)$ were on the lower curtain. Among those lesions, $20.4 \%$ (72 lesions) were located in the frontal lobes, $16.4 \%$ (58) in the temporal lobe, $25.5 \%$ (90) in the parietal lobe, $18.1 \%$ (64) in the occipital lobe, $15.3 \%$ (54) in the basal ganglia and $4.2 \%$ (15) in the cerebellum hemisphere. The average size of lesions was $2.8 \times 3.0 \times 3.0 \mathrm{~cm}$, and most lesions were round or irregular shape.

CT and MRI imaging results. A total of 92 lesions were found from 87 patients who underwent CT examination, and 27 lesions (29.3\%) showed uniform density and 65 lesions (70.7\%) showed high density. Lesions showed clear edges, with low-density edema around the tumor. All lesions showed enhanced signals after enhanced scan. A total of 81 lesions were found from
Table I. Parameter settings for CT and MRI

\begin{tabular}{lcc}
\hline Methods & Thickness $(\mathrm{mm})$ & Layer interval $(\mathrm{mm})$ \\
\hline CT & 10 & 10 \\
MRI & 5 & 1 \\
\hline
\end{tabular}

CT, computed tomography; MRI, magnetic resonance imaging.

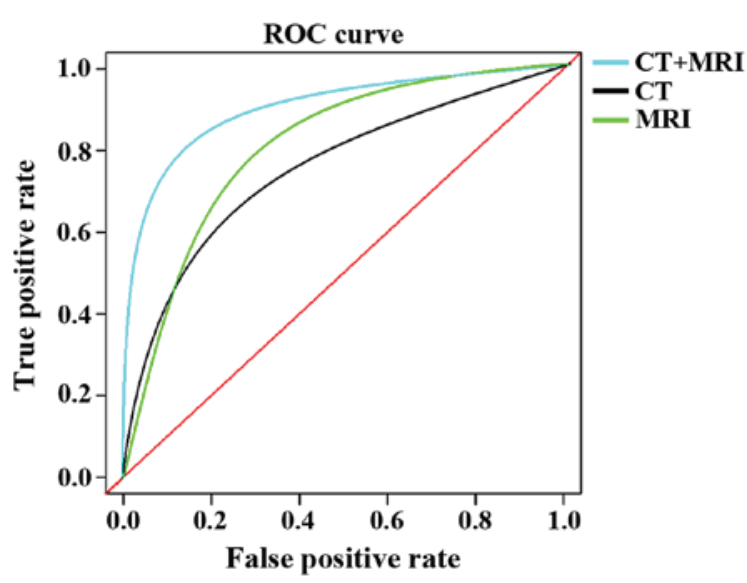

Figure 1. ROC curve analysis. AUC was 0.726 (95\% CI, 0.215-2.337) for MRI; 0.785 (95\% CI, 0.616-1.242) for CT; and 0.845 (95\% CI, 0.145-4.632) for CT combined with MRI. Diagnostic sensitivity and specificity of MRI were 79.3 and $64.9 \%$, respectively. Diagnostic sensitivity and specificity of CT were 75.5 and $67.4 \%$, respectively. Diagnostic sensitivity and specificity of MRI combined with CT were 86.3 and $75.8 \%$, respectively. CT, computed tomography; MRI, magnetic resonance imaging; $\mathrm{CI}$, confidence interval.

74 patients who underwent MRI examination. Results of plain scan showed that all lesions were in uniform state, all lesions showed low signal on T1WI, and $79.0 \%$ (64) lesions showed high signal on T2WI, and $20.2 \%$ (17) lesions showed no signal or equal signal. All lesions showed mild mass effect. After enhanced scan, $86.9 \%$ (73) lesions showed markedly uniform enhancement, $7.4 \%$ (6) showed uneven enhancement, and $2.5 \%$ (2) showed ependymal dissemination. No obvious cystic lesions, calcification or bleeding was found within the lesions.

Diagnostic accuracy. Of the 87 patients who underwent CT examination, 72 cases of primary brain lymphoma were diagnosed, and the diagnostic accuracy was $82.8 \%$. Of 74 patients with MRI examination, 62 cases were diagnosed, and the diagnostic accuracy was $83.8 \%$. Of the 69 patients tested by MRI and CT, 62 cases were detected, and the diagnostic accuracy was $89.9 \%$. Therefore, combined diagnosis showed highest accuracy $(\mathrm{p}<0.05)$ (Table III).

ROC curve analysis. ROC curve analysis showed that the AUC of MRI was 0.726 (95\% CI, 0.215-2.337); the AUC of CT was 0.785 (95\% CI, 0.616-1.242), and the AUC of MRI combined with CT was 0.845 (95\% CI, 0.145-4.632). Diagnostic sensitivity and specificity of MRI was 79.3 and $64.9 \%$, respectively. Diagnostic sensitivity and specificity of CT was 75.5 and $67.4 \%$, respectively. Diagnostic sensitivity and specificity of MRI combined with CT was 86.3 and $75.8 \%$, 
Table II. Clinical patient data.

\begin{tabular}{|c|c|c|c|c|c|}
\hline Variables & CT group $(n=87)$ & MRI group $(\mathrm{n}=74)$ & MRI+CT group $(n=69)$ & F-value & $\mathrm{P}$-value \\
\hline Age (years) & & & & 2.351 & 0.294 \\
\hline$<45$ & $51(58.6)$ & $48(64.9)$ & $39(56.5)$ & & \\
\hline$\geq 45$ & $36(41.4)$ & $26(35.1)$ & $30(43.5)$ & & \\
\hline Sex & & & & 3.250 & 0.348 \\
\hline Male & $57(65.5)$ & $48(64.9)$ & $42(60.9)$ & & \\
\hline Female & $30(34.5)$ & $26(35.1)$ & $27(39.1)$ & & \\
\hline Ethnicity & & & & 3.184 & 0.404 \\
\hline Han & $81(93.1)$ & $70(94.6)$ & $62(89.9)$ & & \\
\hline Others & $6(6.9)$ & $4(5.4)$ & $7(10.1)$ & & \\
\hline Marital status & & & & 2.215 & 0.310 \\
\hline Married & $75(86.2)$ & $61(82.4)$ & $55(79.7)$ & & \\
\hline Unmarried & $9(10.3)$ & $10(13.5)$ & $11(15.9)$ & & \\
\hline Widowed & $3(3.4)$ & $3(4.1)$ & $3(4.3)$ & & \\
\hline Living area & & & & 3.034 & 0.425 \\
\hline Countryside & $59(67.8)$ & $47(63.5)$ & $41(59.4)$ & & \\
\hline Urban area & $28(32.2)$ & $27(36.5)$ & $28(40.6)$ & & \\
\hline Smoking index & & & & 3.076 & 0.352 \\
\hline$<400$ & $45(51.7)$ & $39(52.7)$ & $33(47.8)$ & & \\
\hline$\geq 400$ & $42(48.3)$ & $35(47.3)$ & $36(52.2)$ & & \\
\hline Drinking & & & & 2.585 & 0.316 \\
\hline Do not drink or drink rarely & $48(55.2)$ & $38(51.4)$ & $35(50.7)$ & & \\
\hline Drink a lot & $39(44.8)$ & $36(48.6)$ & $34(49.3)$ & & \\
\hline Exercise habits & & & & 2.489 & 0.259 \\
\hline Yes & $31(35.6)$ & $30(40.5)$ & $26(37.7)$ & & \\
\hline No & $56(64.4)$ & $44(59.5)$ & $43(62.3)$ & & \\
\hline Pathological stages & & & & 3.167 & 0.317 \\
\hline I-II & $44(50.6)$ & $39(52.7)$ & $35(50.7)$ & & \\
\hline III-IV & $43(49.4)$ & $35(47.3)$ & $34(49.3)$ & & \\
\hline Clinical symptoms & & & & 2.529 & 0.277 \\
\hline Headache & $71(81.6)$ & $60(81.1)$ & $55(79.7)$ & & \\
\hline Vomit & $66(75.9)$ & $52(70.3)$ & $46(66.7)$ & & \\
\hline Intracranial hypertension & $54(62.1)$ & $41(55.4)$ & $39(56.5)$ & & \\
\hline Unresponsive & $71(81.6)$ & $65(87.8)$ & $61(88.4)$ & & \\
\hline Abnormal behavior & $43(49.4)$ & $30(40.5)$ & $29(42.0)$ & & \\
\hline Sleepiness & $24(27.6)$ & $25(33.8)$ & $41(59.4)$ & & \\
\hline Cranial nerve paralysis & $39(44.8)$ & $29(39.2)$ & $29(42.0)$ & & \\
\hline Aphasia & $56(64.4)$ & $48(64.9)$ & $48(69.6)$ & & \\
\hline
\end{tabular}

CT, computed tomography; MRI, magnetic resonance imaging.

respectively. The specificity and sensitivity of combined detection were both higher than CT or MRI alone $(\mathrm{p}<0.05)$ (Table IV; Fig. 1).

\section{Discussion}

Primary brain lymphoma is a lymphoma that occurs only in the central nervous system (intracranial) and is not commonly found in intracranial tumors due to its high requirement of conditions of occurrence and development (2). With the development of medical technology, all kinds of technologies that impair the function of the patient's body have been applied and the deterioration of the social environment has changed so that the immune function of the modern human body has plummeted (9). The development of primary brain lymphoma from mononuclear phagocyte system in the perivascular space is due to the lack of immune resistance in the body $(10,11)$. Therefore, in recent years, the incidence 
Table III. Diagnostic accuracy of three methods.

\begin{tabular}{lccc}
\hline Methods & $\begin{array}{c}\text { Diagnosis } \\
(\mathrm{n})\end{array}$ & $\begin{array}{c}\text { Misdiagnosis } \\
(\mathrm{n})\end{array}$ & $\begin{array}{c}\text { Accuracy } \\
(\%)\end{array}$ \\
\hline MRI (n=74) & 62 & 12 & 83.8 \\
CT (n=87) & 72 & 15 & 82.8 \\
MRI+CT (n=69) & 62 & 7 & 89.9 \\
F-value & & & 12.54 \\
P-value & & & 0.036 \\
\hline
\end{tabular}

CT, computed tomography; MRI, magnetic resonance imaging.

Table IV. ROC curve analysis results.

\begin{tabular}{lcccc}
\hline Methods & AUC & $95 \% \mathrm{CI}$ & $\begin{array}{c}\text { Sensitivity } \\
(\%)\end{array}$ & $\begin{array}{c}\text { Specificity } \\
(\%)\end{array}$ \\
\hline MRI & 0.726 & $0.215-2.337$ & 79.3 & 64.9 \\
CT & 0.785 & $0.616-1.242$ & 75.5 & 67.4 \\
MRI+CT & 0.845 & $0.145-4.632$ & 86.3 & 75.8 \\
F-value & & & 11.69 & 12.94 \\
P-value & & & 0.045 & 0.027 \\
\hline
\end{tabular}

CT, computed tomography; MRI, magnetic resonance imaging.

of primary brain lymphoma showed an increasing trend. Studies (12-14) have shown that the onset age of primary brain lymphoma is becoming increasingly younger, which needs additional attention in the clinic. Primary brain lymphoma at the early stages usually shows no obvious signs, and error diagnosis and misdiagnosis are common (15). Imaging is the most commonly used method for the clinical diagnosis of primary brain lymphoma. In this study, the imaging results of 230 patients with primary brain lymphoma were analyzed with an expectation of providing reference and guidance for the early diagnosis of patients with primary brain lymphoma.

In this study, 87 of 230 patients with primary brain lymphoma were examined by CT, 74 by MRI, and 69 by MRI and CT. There was no significant difference in general information among the three groups $(\mathrm{p}>0.05)$. MRI and CT imaging results showed that primary brain lymphoma may occur in any part of the brain. Disease usually develops in the perivascular space between the mononuclear phagocyte system. So, more lesions were found in the intracranial upper curtain (frontal lobe and basal ganglia) than in lower curtain. In this study, the results of CT-examined lesions were mostly isodense or high-density nodules, suggesting that the arrangement of cells in primary brain lymphoma lesions usually shows dense network structure with very little interstitial water and high density of nuclear plasma, so X-ray absorption during the CT scan is quite large, and this was reflected in the imaging results. In MRI examination, imaging results were basically consistent with the relevant studies (16-18); T1W1 showed low signal; T2W2 mostly showed high signal and equal signal, which also proved that the primary brain lymphoma lesions usually showed fibrous mesh arrangment.
Patrick and Mohile (19) suggested that primary brain lymphoma is a malignant tumor that is lesion-centered and destructive to the surrounding area. Therefore, enhanced imaging of MRI is often accompanied by significant enhancement. In this study, tumor scanning by enhanced CT and MRI showed enhanced signal, which supported the mass effects and invasive features of this disease. Therefore, early diagnosis and treatment is needed, in order to avoid the deterioration of primary brain lymphoma. For diagnosis results of CT combined with MRI, diagnosis of primary brain lymphoma is mainly based on meningioma-like melanoma signal. Mild edema, obvious mass effect and big tumor body all meet the imaging features of primary brain lymphoma. Therefore, mass effect may be an indicator of primary brain lymphoma. CT, MRI and combine diagnosis all achieved satisfactory diagnostic results, but are not enough for the qualitative diagnosis of primary brain lymphoma. Therefore, both clinical imaging results and pathological examination results should be used in the diagnosis.

In this study, 230 patients with primary brain lymphoma were studied, and the results of MRI and CT images were analyzed, but there are still some shortcomings. The sample size was small and experimental conditions were limited, and CT and MRI detectors may have differences in image results. We will improve those shortcomings in our future studies.

In conclusion, both CT and MRI can provide satisfactory diagnosis for primary brain lymphoma, but pathological examinations are always needed.

\section{Acknowledgements}

Not applicable.

\section{Funding}

No funding was received.

\section{Availability of data and materials}

The datasets used and/or analyzed during the present study are available from the corresponding author on reasonable request.

\section{Authors' contributions}

YQ and JZ conceived and designed the study. YQ, HL and $\mathrm{XW}$ were responsible for the collection and analysis of the data. AB, GZ and JZ interpreted the data and drafted the manuscript. AB and JZ revised the manuscript critically for important intellectual content. All authors read and approved the final manuscript.

\section{Ethics approval and consent to participate}

The study was approved by the Ethics Committee of People's Hospital of Rizhao (Rizhao, China). Signed written informed consents were obtained from the patients and/or guardians.

\section{Consent for publication}

Not applicable. 


\section{Competing interests}

The authors declare that they have no competing interests.

\section{References}

1. Dolecek TA, Propp JM, Stroup NE and Kruchko C: CBTRUS statistical report: Primary brain and central nervous system tumors diagnosed in the United States in 2005-2009. Neuro-oncol 14 (Suppl 5): v1-v49, 2012.

2. Korfel A, Thiel E, Martus P, Möhle R, Griesinger F, Rauch M, Röth A, Hertenstein B, Fischer T, Hundsberger T, et al: Randomized phase III study of whole-brain radiotherapy for primary CNS lymphoma. Neurology 84: 1242-1248, 2015.

3. Hoang-Xuan K, Bessell E, Bromberg J, Hottinger AF, Preusser M, Rudà R, Schlegel U, Siegal T, Soussain C, Abacioglu U, et al European Association for Neuro-Oncology Task Force on Primary CNS Lymphoma: Diagnosis and treatment of primary CNS lymphoma in immunocompetent patients: Guidelines from the European Association for Neuro-Oncology. Lancet Oncol 16: e322-e332, 2015.

4. Ferreri AJ, Cwynarski K, Pulczynski E, Ponzoni M, Deckert M, Politi LS, Torri V, Fox CP, Rosée PL, Schorb E, et al; International Extranodal Lymphoma Study Group (IELSG): Chemoimmunotherapy with methotrexate, cytarabine, thiotepa and rituximab (MATRix regimen) in patients with primary CNS lymphoma: Results of the first randomisation of the International Extranodal Lymphoma Study Group-32 (IELSG32) phase 2 trial. Lancet Haematol 3: e217-e227, 2016.

5. Nayak L, Pentsova E and Batchelor TT: Primary CNS lymphoma and neurologic complications of hematologic malignancies. Continuum (Minneap Minn) 21: 355-372, 2015.

6. Omuro A, Correa DD, DeAngelis LM, Moskowitz CH, Matasar MJ, Kaley TJ, Gavrilovic IT, Nolan C, Pentsova E, Grommes CC, et al: R-MPV followed by high-dose chemotherapy with TBC and autologous stem-cell transplant for newly diagnosed primary CNS lymphoma. Blood 125: 1403-1410, 2015.

7. Touitou V, LeHoang P and Bodaghi B: Primary CNS lymphoma. Curr Opin Ophthalmol 26: 526-533, 2015.

8. Houillier C, Choquet S, Touitou V, Martin-Duverneuil N, Navarro S, Mokhtari K, Soussain C and Hoang-Xuan K: Lenalidomide monotherapy as salvage treatment for recurrent primary CNS lymphoma. Neurology 84: 325-326, 2015.

9. Omuro A, Chinot O, Taillandier L, Ghesquieres H, Soussain C, Delwail V, Lamy T, Gressin R, Choquet S, Soubeyran P, et al: Methotrexate and temozolomide versus methotrexate, procarbazine, vincristine, and cytarabine for primary CNS lymphoma in an elderly population: An intergroup ANOCEF-GOELAMS randomised phase 2 trial. Lancet Haematol 2: e251-e259, 2015.
10. Vater I, Montesinos-Rongen M, Schlesner M, Haake A, Purschke F, Sprute R, Mettenmeyer N, Nazzal I, Nagel I, Gutwein $\mathrm{J}$, et al: The mutational pattern of primary lymphoma of the central nervous system determined by whole-exome sequencing. Leukemia 29: 677-685, 2015.

11. Yahalom J, Illidge T, Specht L, Hoppe RT, Li YX, Tsang R and Wirth A; International Lymphoma Radiation Oncology Group: Modern radiation therapy for extranodal lymphomas: Field and dose guidelines from the International Lymphoma Radiation Oncology Group. Int J Radiat Oncol Biol Phys 92: 11-31, 2015.

12. Jiang S, Yu H, Wang X, Lu S, Li Y, Feng L, Zhang Y, Heo HY, Lee DH, Zhou J, et al: Molecular MRI differentiation between primary central nervous system lymphomas and high-grade gliomas using endogenous protein-based amide proton transfer MR imaging at 3 Tesla. Eur Radiol 26: 64-71, 2016.

13. Yamada S, Ishida Y, Matsuno A and Yamazaki K: Primary diffuse large B-cell lymphomas of central nervous system exhibit remarkably high prevalence of oncogenic MYD88 and CD79B mutations. Leuk Lymphoma 56: 2141-2145, 2015.

14. Kasenda B, Loeffler J, Illerhaus G, Ferreri AJ, Rubenstein J and Batchelor TT: The role of whole brain radiation in primary CNS lymphoma. Blood 128: 32-36, 2016.

15. Montesinos-Rongen M, Purschke FG, Brunn A, May C, Nordhoff E, Marcus K and Deckert M: Primary central nervous system (CNS) lymphoma B cell receptors recognize CNS proteins. J Immunol 195: 1312-1319, 2015.

16. Mabray MC, Cohen BA, Villanueva-Meyer JE, Valles FE, Barajas RF, Rubenstein JL and Cha S: Performance of apparent diffusion coefficient values and conventional MRI features in differentiating tumefactive demyelinating lesions from primary brain neoplasms. AJR Am J Roentgenol 205: 1075-1085, 2015.

17. Kasenda B, Ferreri AJ, Marturano E, Forst D, Bromberg J, Ghesquieres H, Ferlay C, Blay JY, Hoang-Xuan K, Pulczynski EJ, et al: First-line treatment and outcome of elderly patients with primary central nervous system lymphoma (PCNSL) - a systematic review and individual patient data meta-analysis. Ann Oncol 26: 1305-1313, 2015.

18. Welch MR, Sauter CS, Matasar MJ, Faivre G, Weaver SA, Moskowitz CH and Omuro AM: Autologous stem cell transplant in recurrent or refractory primary or secondary central nervous system lymphoma using thiotepa, busulfan and cyclophosphamide. Leuk Lymphoma 56: 361-367, 2015.

19. Patrick LB and Mohile NA: Advances in primary central nervous System lymphoma. Curr Oncol Rep 17: 60, 2015.

This work is licensed under a Creative Commons

Attribution-NonCommercial-NoDerivatives 4.0 International (CC BY-NC-ND 4.0) License. 